\title{
Analysis on characteristics of Earthquake in the West Chinese Mainland and Its Adjacent Areas
}

\author{
Shao Huicheng ${ }^{1,2, a}$, Fu Zhengxiang ${ }^{3, b}$ \\ ${ }^{1}$.Department of Mechanics, Northwestern Polytechnical University, 710072 Xi'an, China \\ 2. Earthquake Administration of Shaanxi Province, 710068 Xi'an, China \\ 3. Institute of Earthquake Science, CEA, 100036 Beijing, China \\ a.shaohcsn@126.com; b. fzx@seis.ac.cn
}

Keywords: WCAA; Wavelet; LOD; Earthquake activity; Time-variant

Abstract: Annual relaxation of the seismic energy between 1900 and 2010 in West Chinese Mainland and Its Adjacent Areas has been deal with wavelet method. The active period of 46, 24, 15 and 7 year in seismic activity have been found by this method. This result, that there are 4 main period in seismic activity, is statically although the results have small difference when the data of the different time term have been used. It would be suggested that the boundary effect of the results should be considered when we make the earthquake prediction for the future seismic tendency. At same time the relation between the five years frequency for $M \geqq 5$ from 1970 in western China and LOD also found. Two years after the turning of LOD is high occurrence for $M \geqq 5$.

\section{Introduction}

The seismic activity there is Alternating of activity exists and quiescence periods, has been confirmed by many studies [1,2]). Distinguishing the high and low seismic activity periods in Chinese mainland is of great importance for guiding earthquake forecast of mid-short term, especially for short term analysis and prediction of the strong earthquakes, and is an indispensable step for the study of earthquake prediction in China $[3,4,5]$. The earthquake activity is suddenly evolved from one state to another, which we call as abrupt change. Sudden change is a very important and common characteristic of non-linear system.

West Chinese Mainland and Its Adjacent Areas (WCMAA) is one of the most active seismic areas in the world. In this paper, we use the wavelet to analysis the seismic Characteristic of WCMAA.

\section{Wavelet Transforms and Abrupt Change Analysis}

If $\psi(t)$ is the second derivative of a smooth function, $\psi(x)=d 2 \theta(x) / d 2 x$, then zero point for wavelet transformation is turning point of the function, and it is also ladder point in extreme situation $[6,7]$.

$$
\begin{aligned}
& W_{f(}(a, b)=\left(W_{\psi f} f\right)(a, b)=W f(a, b) \\
& =f(x) * \psi_{a, b}^{*}(x)=a^{2} \frac{d^{2}}{d x^{2}}\left[f * \theta_{a}^{*}(x)\right] \quad a>0, f \in \mathrm{L}^{2}(\mathrm{R}) .
\end{aligned}
$$

where * refers complex conjugate.

Formula (1) can be seen as the smooth result of the signal by the wavelet basic function with the scale, in which parameter $a$ is lengthen and shorten factor, $b$ is position factor. The zero point of the wavelet transformation is just turning point of the function and the abrupt point in the extra situation $[8,9]$.

$$
\psi(t)=\pi^{-1 / 4} e^{-\mathrm{i} \omega \mathrm{t}} e^{-\mathrm{t}^{2} / 2} \quad \omega \geq 5 .
$$

Morlet wavelet (2) is complex number and it has the best differentiated ratio in time and frequency field and can get breadth value and phase information of time process or signal analyzed object, so the wavelet is usually used in the study of geophysics process and swift flow analysis. It can be proved that the relation between scale parameter a and Fourier period $\mathrm{T}$ is $\mathrm{T}=1.03 \mathrm{a}$ as $\omega=6[10$, 
11]. Thus, enduing the scale factor with some physical meaning, we can change wavelet spectrum to frequency spectrum with physical meaning. By transmitting the scale parameter a and translation parameter $b$, we can acquire the frequency of time sequence at any time and its variable character at any time-frequency field $[6,7,12]$.

\section{Cycle Analysis of Seismic Activity of WCMAA}

In recent years, West Chinese Mainland and Its Adjacent Areas (WCMAA) have occurred Sep.27, 2003 Russia and Mongolia, Oct. 8, 2005 Pakistan 7.8 and the May. 12, 2008 Wenchuan and Apr. 14, 2010 Yushu 7.1 earthquakes, these earthquake caused a great disaster.

Engdahl et al in 2002 to collect, collate different regions, different versions of the catalog, based on the compilation of the twentieth century compiled a comprehensive global seismic activity and earthquakes from the appropriate directory. Analysis of the WCMAA in order to better seismic activity features to the earthquake characteristic, added Harvard University CMT catalog, the "Triangle" area of seismic activity. Figure 1 shows the 1900 to 2010 the energy released in an earthquake sequence in the region of the Morlet wavelet transform results. Seismic activity mainly in the 46, 24, 15 and 7 events in four major periods, reflect the variation of WCMAA area on the release of seismic energy in time-frequency domain.
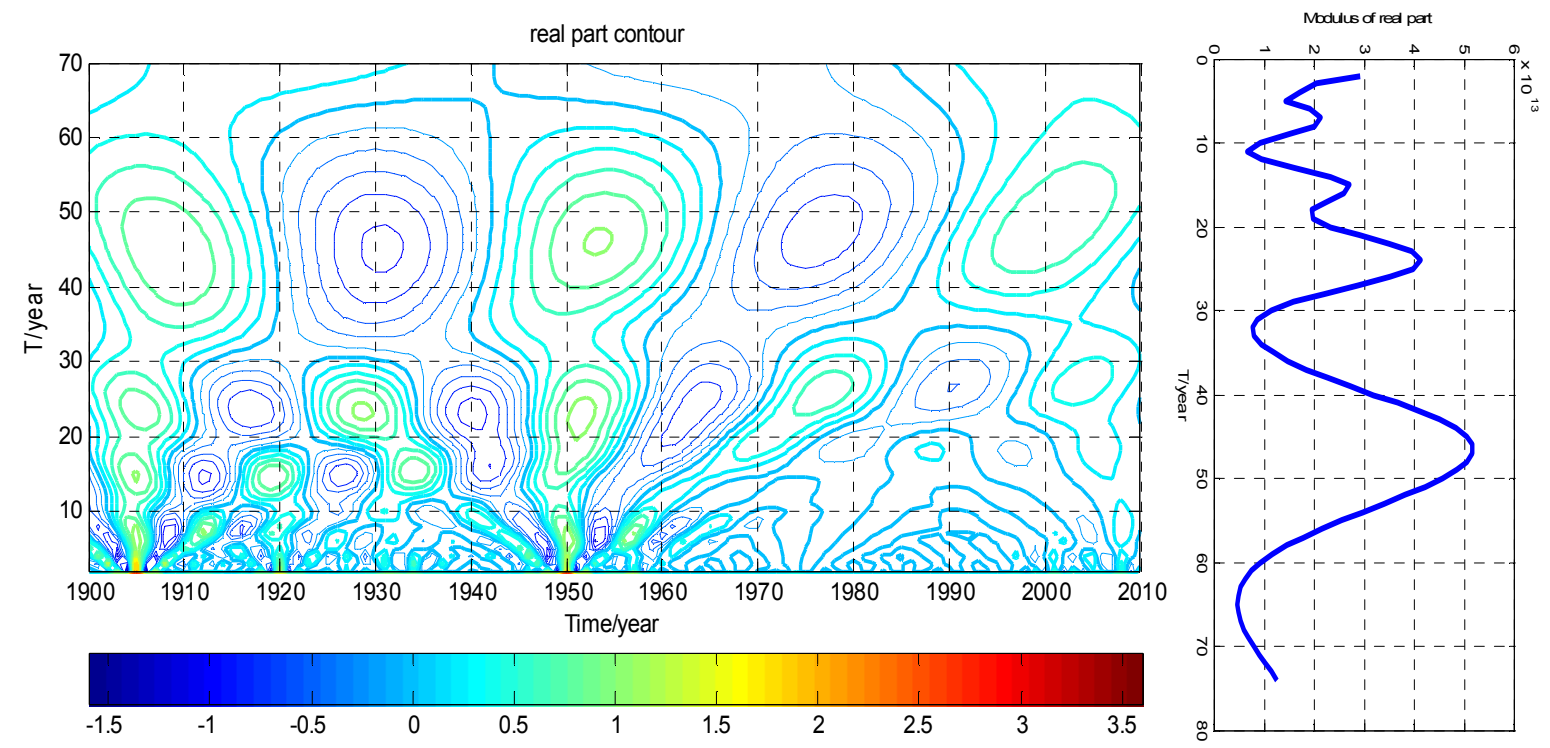

Fig1. Isograms of actual part of wavelet coefficient for annual seismic energy relaxation

In 46 years, 24 years, 15 years and 7 years or so there are more obvious on the scale of the mutation characteristics is, alternating strong and weak seismic activity. Wavelet coefficients from the main activity cycle profile can be seen that certain of its intensity changes with time, that "the variability", such as 15-year period to the performance in 1960 was significantly better than the last century(Fig.2).

Figure 3 shows, respectively, using the 1900 to 2000 and 1900 to 2010 time period of two data cycles are the main results of seismic activity.

It can be seen from Figure 3, the application of information at different times, have concluded there are some differences. This difference is actually a wavelet transform edge effect. Thus, the seismic wavelet coefficients of trend prediction, we must consider this effect. 

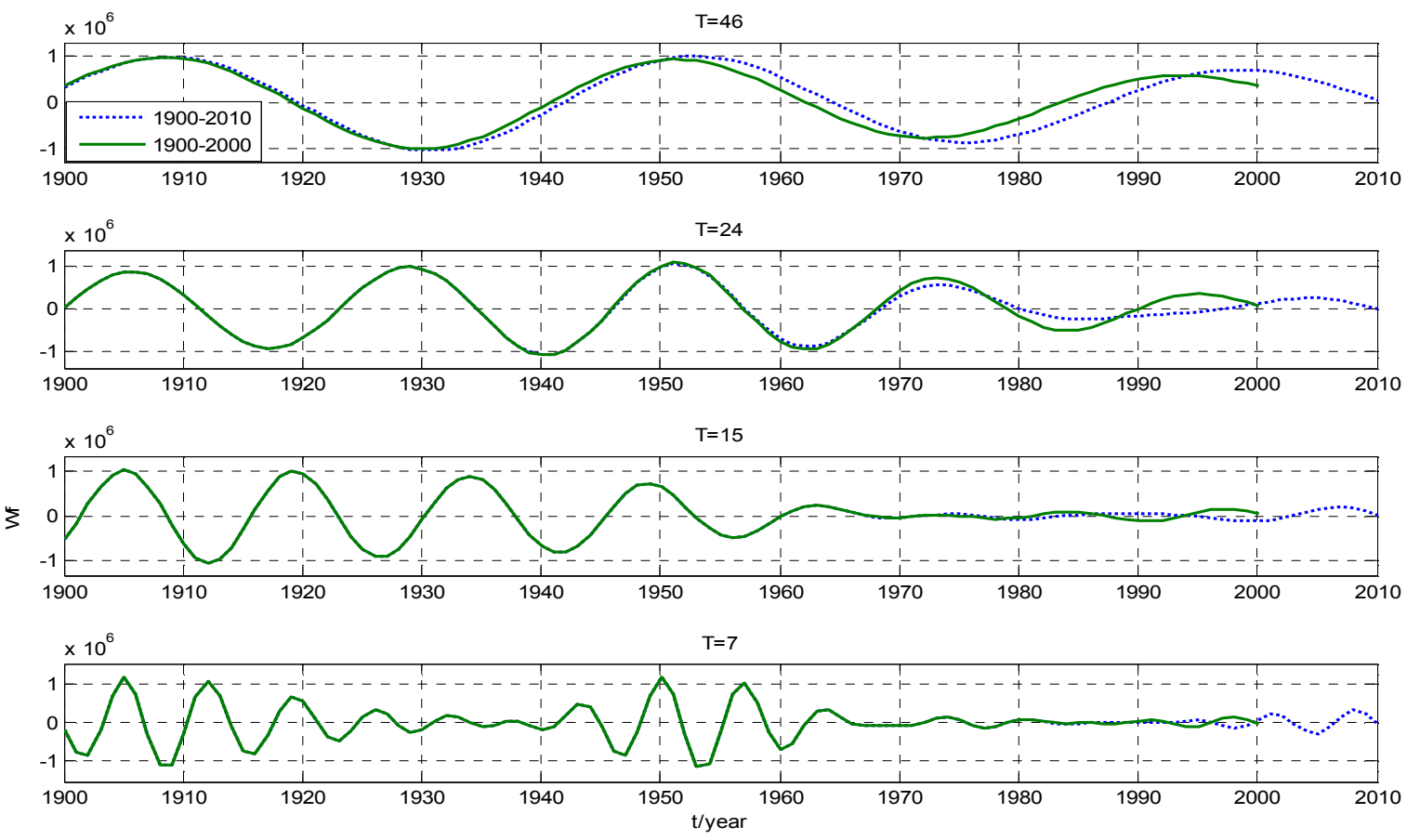

Fig.2 Actual part of wavelet coefficient of 4 main active period

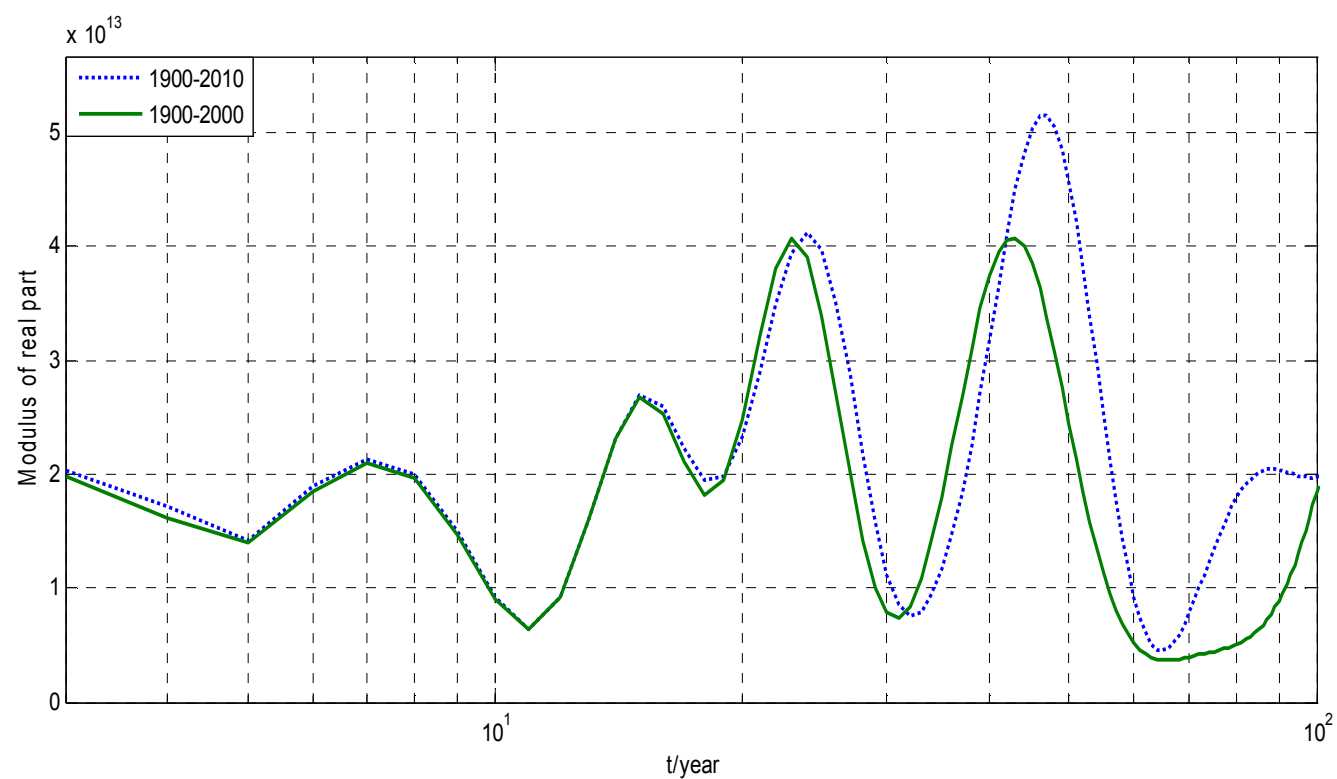

Fig.3 Comparison the results of different data periods

\section{Shallow earthquakes in West Chinese Mainland relations with the Earth's rotation}

Global and regional tectonic features of spatial and temporal distribution of seismic activity and earth rotation relationship between the non-uniformity study was the concern of many scientists at home and abroad. Uniform rotation of the earth movement that provided the strain energy, attached to the full strain energy has accumulated on the fault, can trigger seismic activity $[13,14]$.

Previous results show that in 1950, West Chinese Mainland 5 earthquakes are relatively complete. In order to better reflect the rhythm of seismic activity, Figure 4 shows the adjacent areas in western China and $M \geq 5$ earthquakes window length of 3 years and 5 years and 7 sliding on the frequency of earthquakes and the LOD diagram. Can see that since 1970, five earthquakes slip 
frequency and length of the day there is a certain consistency of changes, including the frequency of seismic activity 3 times on high-value segment and the Earth's rotation from slow to fast transition time corresponding to 2 times the local high Point and the Earth's rotation from fast to slow transition time corresponding to the lag of about 2 years. Therefore, turning the Earth's rotation rate variation of more than five western China earthquakes triggered a certain role.
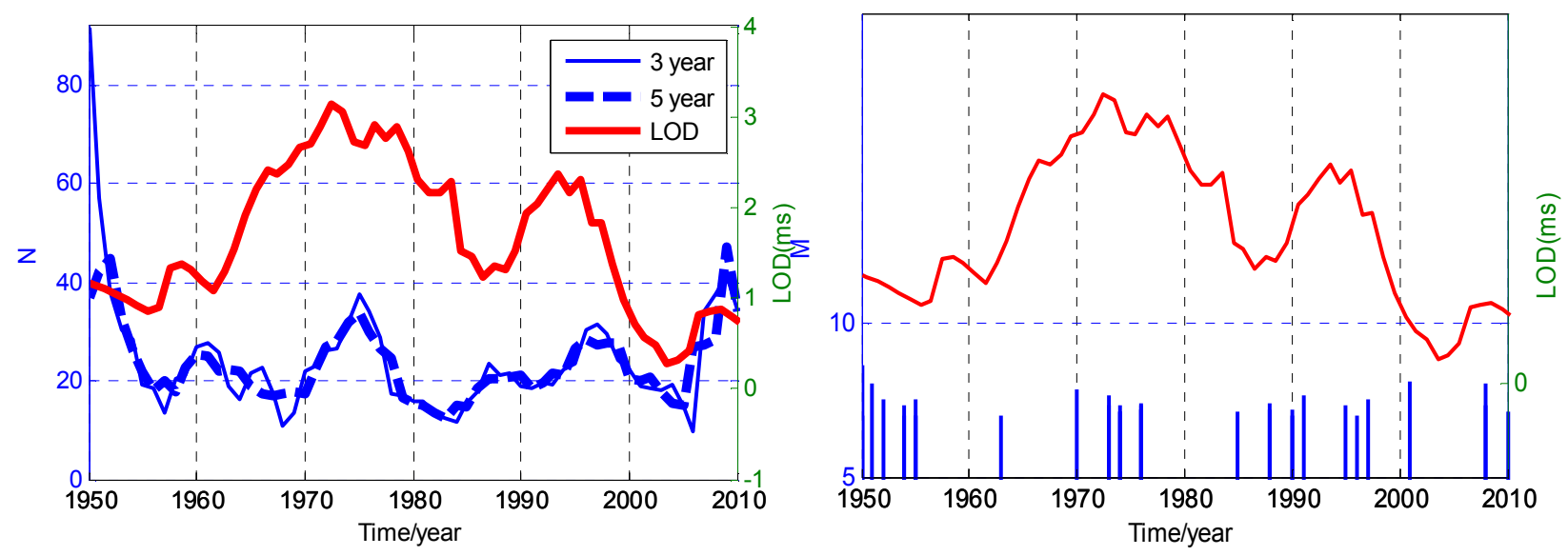

Fig 4. Relationship between 5 earthquakes in frequency (left) and 7 earthquake (right) with

Although the sample of more than magnitude 7 earthquakes less than magnitude 7 earthquake in MT but the figure also shows that more than magnitude 7 earthquakes occurred in the Earth's rotation rate variation transition period. Seventies of last century in particular, the mainland is indeed time the climax of seismic activity since the last century is a major trend in the Earth's rotation changes the turning point. Turning point in the last century the Earth's rotation period is more than magnitude 7 earthquake in the mainland with multiple stages, but there is no centralized seventies of last century.

\section{Results and Discussion}

Wavelet analysis at different time scales changes in the pattern of seismic activity, seismic activity for the staging of a new way of thinking.

1. Western China and adjacent areas there is significant seismic activity 46 -year activity cycle, 24,15 , live in harmony-year cycle in the performance of the first half of last century, significant features of the subsequent cycle is not obvious.

2. data from different time periods the results obtained show that the wavelet transform coefficients obvious edge effect, trend forecasting based on wavelet coefficients, we must consider this effect.

3. 1960 years, more than five western China earthquake frequency and rate of change in the earth there are some autobiographical relevance.

\section{Acknowledgments}

We thank Professor Jin Xueshen for help. This research was supported by the project "The study of Earthquake Risk in Northern of Chinese North-South Seismic Belt" of CEA and Joint Earthquake Science Foundation (A07109). 


\section{References}

[1] L. Mogi, Active periods in the world's chief seismic belts. Tectonophysics, 22(1974)265-282.

[2] Z.J. Ma, The global seism tectonic systems, Earth Science-Journal of Wuhan College of Geology, 18(3) (1982)23-38.

[3] G.M. Zhang, L. Li, Scientific Thought of Prediction of Earthquake and Trend in the Coming Years in China, In: Center of analysis and prediction of China Seismological Bureau, Study earthquake trend in China(1998). Beijing: Seismological Press, 174-187, 1997 (in Chinese).

[4] G.M. Zhang, J. Liu, Y.L Shi, An scientific evaluation of annual earthquake prediction ability, Acta Seismologica Sinica, 15(5) (2002)550-558.

[5] Z.X. Fu, J. Liu, G. P. Liu, Three Division Method of Clustering Strong Earthquake Activity in China's Continent and its Application, Earthquake, 20(2000)Sup. 34-37 (in Chinese).

[6] H.C. Shao, C.E. Du, Z.H. Liu, Y.X. Sun, C.Q. Xia, Multi-scale analysis of earthquake activity in Chinese mainland, Acta Seismologica Sinica, 17(1) (2004)109-113.

[7] H. C. Shao, X.X. Du, X.S. Jin, C.E. Du. The Application of the Wavelet Analysis in Earthquake Prediction, Earthqyake Research in China, 16(1) (2000)48-52(in Chinese).

[8] T.Z. Liu, P.P. Rong, S.D. Liu. et al. Wavelet Analysis of Climate Jump, Chinese J. of Geophysics, 38(2)(1995)158-162(in Chinese).

[9] F.S. Yang, Analysis and Application of Wavelet Transformation in Engine, Beijing: Science Press, 2001(in Chinese).

[10] J.P. Laura, D.N. David, Wavelet Analysis of Velocity Dispersion of Elastic Interface Waves Propagating Along a Fracture. Geophys Res Lett, 22(11) (1995)1329-1332.

[11] Y.X. Xu, J.Y. Wang, Power Spectrum Estimation for Magnetotelluric Signal Based on Continuous Wavelet Transform, Chinese J. of Geophysics 43(5)(2000)677-683(in Chinese).

[12] X.X. Du, Wavelet-based Analysis of Dynamic Seismicity Period, Earthqyake, 17(3)(1997) 257-264(in Chinese).

[13] Z.X. Fu, H.C. Shao, X. Ding, Relation between distribution of shallow strong earthquake in china's continent and the earth rotation rate variation, Earthquake, 24(3)(2004)15-20(in Chinese).

[14] W.J. Bo, G.Y. Wang, Research on relations among earthquake rotation, Fault deformation and seismicity, Journal of Geodesy and Geodynamics, 26(2)(2007)43-47(in Chinese) 